\title{
Polymethylmethacrylate-augmented Cannulated Pedicle Screw Fixation for High-energy Vertebral Compression Fracture With Osteoporosis
}

\section{Yao-yao Liu}

Third Military Medical University Daping Hospital and Research Institute of Surgery Jun Xiao

PLA Rocket Force University of Engineering

\section{Xiang Yin}

Third Military Medical University Daping Hospital and Research Institute of Surgery

\section{Ming-Yong Liu}

Third Military Medical University Daping Hospital and Research Institute of Surgery

\section{Zhong Wang}

Third Military Medical University Daping Hospital and Research Institute of Surgery

Lei He

Third Military Medical University Southwest Hospital

\section{Peng Liu}

Third Military Medical University Daping Hospital and Research Institute of Surgery

Fei Dai ( $\nabla$ david-feifei@163.com )

Army Medical University

\section{Research}

Keywords: Osteoporosis, compression fracture, pedicle screw, bone cement

Posted Date: May 5th, 2021

DOI: https://doi.org/10.21203/rs.3.rs-482277/v1

License: (1) (1) This work is licensed under a Creative Commons Attribution 4.0 International License. Read Full License 


\section{Abstract}

Background: High-energy vertebral compression fracture (HVCFs) with osteoporosis has higher requirement for the stability of pedicle screw internal fixation system. However, few studies have involved the clinical outcome of cannulated pedicle screw to augment fixation with polymethylmethacrylate (PMMA) under this condition. This study aims to investigate the mid-term efficacy of bone cementinjectable cannulated pedicle screw (CICPS) in HVCFs with osteoporosis.

Methods: Eighteen patients with HVCFs ( $T<-2.5)$ were underwent spinal fixation by CICPS from 2012 to 2017. The operation time, blood loss, and hospitalization time were recorded. Pain and functional recovery were evaluated by Visual Analog Scale VAS) and Oswestry Disability Index (ODI), respectively. Xray films were taken to evaluate the loss of vertebral height, kyphosis angle, pedicle screw loosening, and bony fusion. Surgical related complications were also recorded.

Results: The average follow-up time was $18.5 \pm 8.7$ months (range, 6-54 months). VAS and ODI scores indicated significant improvements in postoperative pain and activity function $(P<0.05)$. Comparing the last follow-up with the postoperative imaging findings, the loss of vertebral height and kyphosis angle was $3.4 \pm 1.2 \mathrm{~mm}$ and $7.8 \pm 3.8^{\circ}$, respectively. The rate of bone graft fusion was $100 \%$ while no cases were involved in screw loosening or extraction. 1 case of superficial infection, 2 cases of PMMA leakage, and 1 case of cerebrospinal fluid leakage were found.

Conclusions: CICPS fixation using PMMA augmentation may suggest as a feasible surgical technique in osteoporotic patients with HVCFs.

\section{Introduction}

The incidence of osteoporotic fractures is increasing significantly with a rapidly ageing population [1], especially among postmenopausal women, the data is up to 50\% [2]. Vertebral compression fractures (VCFs) are the most common condition $[3,4]$ and a major socio-economic burden for elderly populations, which widely performed in the treatment of percutaneous vertebroplasty (PVP) and kyphoplasty (PKP). Up to now, these two minimally invasive surgical methods have been proved to have an excellent clinical effect $[5,6]$.

However, vertebral compression fractures due to high-energy trauma (HVCFs), which defined as VCF combined with severe clinical manifestations such as spinal nerve compression, spinal stenosis, instability, delayed kyphosis and neurological impairment (Kümmell's disease),need to restore spinal stability by internal fixation and decompress spinal canal to relieve the neurological symptoms. The challenge to surgeons is that osteoporosis tends to cause screw loosening and trabecular fracture [7]. Loss of vertebral height, pseudoarthrosis and bone graft non-fusion, followed by inadequate holding power of internal fixation, ultimately may lead to chronic lower back pain, kyphosis and dysneuria. 
Studies have shown that cannulated pedicle screw augmented by polymethylmethacrylate (PMMA) can significantly improve the screw holding power [8-10] .However, few studies involve the clinical outcome of cannulated pedicle screw to augment fixation in VCF with osteoporosis due to high-energy trauma. Authors improved the design and created a new type of PMMA-augmented pedicle screw named bone cement-injectable cannulated pedicle screw (CICPS). To evaluate the clinical results and feasibility of this technique in HVCFs with osteoporosis, we retrospectively reviewed the data of eighteen consecutive patients treated with CICPS, and summarized the incidence of complications and the clinical efficacy of PMMA augmentation.

\section{Materials And Methods 2.1 Study design}

The study was approved by the Daping Hospital ethics committee (IRB, 2019149); and informed consent was obtained from each participant. This study was a retrospective investigation of osteoporotic patients who underwent surgery at our clinic for HVCFs. Considering that using conventional pedicle screws in cases with a severely osteoporotic vertebral body may lead to screw loosening or pulling out, it would be unethical to include a control group for comparison. Instead, we evaluated the clinical outcomes of this technology by comparing the baseline with post-treatment measurement parameters.

\subsection{Patients}

From October 2012 to May 2017, a total of 30 cases with HVCFs were collected. Inclusion criteria were as follows: (1) Diagnosed as osteoporosis by dual-energy X-ray absorptiometry (T-value <-2.5) [10]; (2) HVCFs with acute or chronic impairment of spinal cord function, spinal stenosis or spinal structural instability; (3) Used CICPS for internal fixation; (4) consented the treatment requirement and schedule. Exclusion criteria were as follows: (1) Simple fragility fracture; (2) Old fracture; (3) Not used CICPS for internal fixation; (4) Allergy to any implant or having surgical contraindications. Figure 1 shows the flow chart of patient recruitment and study procedures.

\subsection{Materials}

The preliminary clinical application of CICPS had achieved satisfactory results in the previous studies performed by authors [8-10]. In references 8 to 10, we had described the design features of CICPS in detail. It is designed as a hollow rod with three side holes at the screw tip so that PMMA can outflow into the centrum through this route. Fixation ability is augmented because of anchoring the screw into cancellous bone with PMMA. In this study, the diameter and length of CICPS were determined according to the measurement results during actual intraoperative conditions. PMMA was a high-viscosity bone cement supplied by Heraeus Holding, Germany.

\subsection{Surgical techniques and postoperative treatment}


The screws were manually placed according to the anatomical landmarks. PMMA powder and water agent were mixed, and then, injected by a special device during a dough-like mass viscosity. No more than $2 \mathrm{ml}$ of PMMA mixture was determined by intraoperative monitoring of intravertebral PMMA dispersion. The following measures were taken to ensure the augmentation effect and further to reduce the PMMA leakage risk: 1. CICPS suggested to keep parallel to the upper and lower vertebral endplates; 2 . The probe was used to directly detect the required screw length so that the outflow side hole of CICPS was located in the anterior 2/5th of the vertebral body; 3 . CICPS was not allowed to penetrate the upper, lower, inner and outer sidewalls of the pedicle and the lateral cortex of the vertebral body; and 4 . The entry angle of CICPS was slightly larger than that of a conventional pedicle screw [9]. The quantity of CICPSs was determined by the bone quality, the stability requirement, and manual feeling during the tunnel preparation. In principle, a minimum quantity of CICPSs was recommended due to the difficulty in removing and repairing. The remaining screws adopted conventional ones. Decompression, fixation and fusion was performed as usual, if necessary.

Antibiotics were routinely used to prevent surgical site infection in the first 48 hours, and the wound drainage tube was removed after 48-72 hours after the amount of drainage fluid was less than $50 \mathrm{ml}$. Three days after the surgery, patients were encouraged to perform rehabilitation exercises by wearing a thoracolumbar brace. Anti-osteoporosis treatment should be supplemented as soon as possible after operation.

\subsection{Safety and effectiveness evaluation}

Operation time, blood loss, and hospitalization time were recorded to evaluate the basic condition of the surgery. A review was carried out at 3 months, 6 months, 1 year, and every half-year after surgery. Moreover, lumbar X-ray films were obtained to evaluate bony fusion, loss of vertebral height, loss of the Cobb angle, and screw loosening or pull-out. Visual Analog Scale (VAS) and Oswestry Disability Index (ODI) scoring systems were used to evaluate pain and functional recovery in lower limbs, respectively. Complications such as wound infection, cerebrospinal fluid leakage, and PMMA leakage were recorded.

The method of measuring the loss of vertebral height: The vertebral body height measured in sagittal Xfilm was used to evaluate fracture reduction. Distances were measured between the following points: the most dorsal superior and inferior endplate margins, the most anterior superior and inferior margins, and the height of the centrums from the midpoint of the two lines. The average values were calculated, and vertebral height was determined after taking into consideration the change in vertebral height between the postoperative review and the last follow-up that was regarded as the value of vertebral height loss [10].

\section{The method of measuring the Cobb angle}

The Cobb angle was used to assess sagittal deformity on a plain radiograph. The upper and lower vertebral bodies near the fractured part with the largest inclination angle were considered, and the angle between the laminae was measured as the kyphosis Cobb angle. The angle change between the postoperative review and the last follow-up was regarded as the value of Cobb angle loss [10]. 
Evaluation criteria for spinal fusion on X-ray films [10,11]: (1) Passage of trabecular bone through the bone graft area; (2) Vertebral movement between flexion and extension X-ray film $<3 \mathrm{~mm}$ or change in the intervertebral space angle $<5$ degrees; and (3) Bone growth through the intervertebral disc space.

\section{Evaluation criteria for screw loosening on X-ray films}

(1) Screw displacement on a lateral X-ray greater than $1 \mathrm{~mm}[12,13]$; (2) Double-circle sign around the screw on an anterior-posterior X-ray film [14].

\subsection{Statistical analysis}

The experimental data were calculated as mean \pm standard deviation $(\mathrm{x} \pm \mathrm{s})$ and analyzed by using the SPSS 13.0 statistical software package. The paired test was conducted to compare variables, including VAS and ODI scoring and imaging indicators at the pre-operation, post-operation, and at the last followup. The change was considered statistically significant if $p$ was less than 0.05 .

\section{Results}

Twenty cases were enrolled and the follow-up rate at each time point was $90 \%$ because 2 cases were lost at three months after operation. Finally, 18 cases were completely recorded for an average follow-up time of $18.5 \pm 8.7$ months (range, $6-54$ months). These cases comprised 6 males and 12 females with an average age of $72.3 \pm 6.5$ years ranging from 56 to 80 years. The average duration from injury to admission was $20 \pm 13.5$ days (range, 3 hours to 3 months).

The operation time was $165 \pm 23 \mathrm{~min}$ (range, 95-462 min), the bleeding amount was $206 \pm 75 \mathrm{ml}$ (range, $50-400 \mathrm{ml}$ ) and the length of the hospitalization time was $12.5 \pm 3.5 \mathrm{~d}$ (range, 9-22 d). There was no death, surgical related neurological dysfunction and paraplegia in all cases at each follow-up time point. One case of superficial surgical site infection was noted, which was under control after an intravenous drip of antibiotics. Cerebrospinal fluid leakage occurred in one case, which healed completely after the drainage tube removed following two week of bed rest. With respect to PMMA leakage, there was one case leakage into the paravertebral vein, and another case into the intervertebral disc space during the operation, but no serious complications such as nerve or blood vessel injury and pulmonary embolism were observed (Table 1).

Table-1 Baseline characteristics and clinical parameters of the 18 patients with high-energy vertebral compression fracture (HVCFs) 


\begin{tabular}{|c|c|}
\hline Variable & Value \\
\hline Average age $(\mathrm{Y})$ & $72.3 \pm 6.5(56-80)$ \\
\hline Height $(\mathrm{cm})$ & $162.3 \pm 5.9(149-175)$ \\
\hline Weight $(\mathrm{kg})$ & $50.3 \pm 12.3(44-78)$ \\
\hline Follow-up time (M) & $18.5 \pm 8.7(6-54)$ \\
\hline Pre-hospital time $(\mathrm{d})$ & $20 \pm 13.5(3 h-3 M)$ \\
\hline Gender (male: female) & $6: 12$ \\
\hline Average BMD (T-value) & $-3.20 \pm 0.57(-2.5 \rrbracket-5.2)$ \\
\hline \multicolumn{2}{|l|}{ Diagnosis } \\
\hline HVCFs with spinal stenosis & $9 / 18(50 \%)$ \\
\hline HVCFs with kyphosis & $4 / 18(22.2 \%)$ \\
\hline HVCFs with delayed kyphosis and neurological impairment & $5 / 18(27.8 \%)$ \\
\hline Operation time $(\mathrm{min})$ & $165 \pm 23(95-462)$ \\
\hline Blood loss (ml) & $206 \pm 75(50-400)$ \\
\hline Hospitalization time (d) & $12.5 \pm 3.5(9-22)$ \\
\hline Number of loosening CICPSs / total number of CICPSs used & $0 / 40$ \\
\hline \multicolumn{2}{|l|}{ Complication } \\
\hline Superficial infection & $1 / 4(25 \%)$ \\
\hline PMMA leakage & $2 / 4(50 \%)$ \\
\hline Cerebrospinal fluid leakage & $1 / 4(25 \%)$ \\
\hline Fusion rate & $100 \%$ \\
\hline
\end{tabular}

\subsection{Imaging evaluation results}

The height of the compressed vertebral body and the kyphosis angle were significantly improved $(\mathrm{P}<$ 0.05). The vertebral height changed from $16.3 \pm 5.6 \mathrm{~mm}$ before operation to $22.7 \pm 4.1 \mathrm{~mm}$ after operation, and to $19.3 \pm 3.9 \mathrm{~mm}$ at the last follow-up; the kyphosis angle changed from $35.6 \pm 10.4$ degree to $18.5 \pm 8.9$ degree, and to $26.3 \pm 9.3$ degree at these three time points. At the last follow-up, the average values of vertebral height were decreased and kyphosis angle were increased at the same time. However, comparing the date at post-operation with that of last follow-up, the vertebral height was no significant change $(P>0.05)$ while the loss of kyphosis angle changed significantly $(P<0.05)(T a b l e-2)$. No screw loosening and fusion failure was observed in all cases according to the criteria. 
Table-2 Height of the compressed vertebral body, Cobb angle, VAS and ODI score in patients before and after treatment.

\begin{tabular}{|llll|}
\hline Index & Pre-operation & Post-operation & Last follow-up \\
\hline Vertebral height $(\mathrm{mm})$ & $16.3 \pm 5.6$ & $22.7 \pm 4.1$ & $19.3 \pm 3.9^{\mathrm{b}}$ \\
\hline Cobb angle (degree) & $35.6 \pm 10.4$ & $18.5 \pm 8.9$ & $26.3 \pm 9.3^{\mathrm{a}}$ \\
\hline VAS score & $8.3 \pm 2.6$ & $2.2 \pm 0.8$ & $1.8 \pm 0.3^{\mathrm{b}}$ \\
\hline ODI score (\%) & $75.5 \pm 12.2$ & $35.2 \pm 7.2$ & $30.5 \pm 13.1^{\mathrm{b}}$ \\
\hline
\end{tabular}

Note: All indexes at the post-operation and last follow-up showed significant differences from those at the pre-operation, $p<0.05$. $a, p<0.05$, a significant difference was observed in Cobb angle between the post-operation and the last follow-up. $b, p>0.05$, no significant difference was observed in the vertebral height, VAS score, and ODI score between the post-operation and the last follow-up.

\subsection{Pain relief and functional recovery}

The VAS score improved from $8.3 \pm 2.6$ before operation to $2.2 \pm 0.8$ after operation, and to $1.8 \pm 0.3$ at last follow-up. The ODI score improved from $75.5 \pm 12.2 \%$ to $35.2 \pm 7.2 \%$, and to $30.5 \pm 13.1 \%$ at these three time points. These indexes were significantly improved after operation $(P<0.05)$, but no significant difference was observed comparing post-operation with last follow-up ( $P>0.05)$ (Table-2).

\subsection{Typical cases}

Typical case 1 (Fig. 2): A 68-year-old female patient (T-value = -3.0), who had suffered from traumatic fracture of the T12 vertebral body 2 months ago, was diagnosed as HVCF and kyphosis, and she did not receive any bed rest and brace treatment. Imaging examinations showed wedge-shaped deformation of the T12 vertebral body and thoracolumbar kyphosis (Fig. 2A-D). Thus, CICPS was used to strengthen the internal fixation, and the kyphosis was corrected after distraction and reduction (Figs. 2E and F). Pouring PMMA with PVP device was performed to maintain vertebral height. 6 and 12 months after operation, bony fusion was achieved with maintenance of the spinal curvature and vertebral height, and there was no PMMA displacement or screw loosening (Fig. 2G-J).

Typical case 2 (Fig. 3): A 73-year-old female patient (T-value = -3.5) suffered from L1 HVCF, kyphosis, and cauda equina injuries due to a fall one month ago (Fig. 3A-D). She was treated with spinal canal decompression, posterolateral arthrodesis, and CICPS-augmented internal fixation of upper and lower vertebral bodies (Fig. 3E and F). The symptoms were then relieved after operation and CICPS loosening was not detected at the 18-month follow up. Although the vertebral height was reduced, the kyphosis angle was maintained and bony fusion was successfully achieved (Fig. 3G and H). PMMA was leaked into the anterior venous plexus at L1 without any symptom. 


\section{Discussion}

Known as a microinvasive technology, PVP and PKP, is considered as the "gold standard" for the treatment of osteoporotic VCFs $[5,6,15]$. However, PVP and PKP may no longer be applicable when VCFs accompanied with severe high-energy trauma. Some special reasons are as follows: 1. High-energy trauma may cause spinal canal stenosis due to the vertebral posterior wall fracture. Symptoms of neurological impairment usually occur which requiring spinal decompression and fixation [16, 17]. 2 . Internal fixation must be performed to restore spinal stability based on the three-column fracture due to high-energy trauma. 3. Delayed neurological symptoms, causing by local kyphosis and wedging of the injured vertebral, require kyphosis correction by spinal fixation [18], and even require support to the anterior and middle columns [19]. However, when internal fixation is performed, osteoporosis seriously reduces the stability in HVCFs patients. Studies have documented that fixation stability in osteoporotic spine reduced significantly with a loosening rate up to $12 \%$ [20].

Up to now, PMMA-augmented cannulated pedicle screw is considered an optional technique which has broad application prospects $[21,22]$. Some researchers recommended this type of pedicle screw for lumbar spondylolisthesis with osteoporosis [23, 24]. Meanwhile, Sawakami et al. [25], Park et al. [26] and Cho et al. [27] have also achieved satisfactorily clinical efficacy in pseudoarthrosis following VCFs and Kummell's disease. But few studies have involved the clinical outcome of PMMA-augmented cannulated pedicle screw in HVCFs. CICPS is a new type of PMMA-augmented pedicle screw created by research group. Previous in vitro study showed that CICPS could significantly improve the biomechanical stability of pedicle screws, and preliminary clinical application suggested that CICPS had satisfactory reliability and safety in osteoporotic patients with lumbar spondylolisthesis and thoracolumbar VCF (AO Type A) $[8-10,28]$.

In current study, the VAS and ODI score significantly improved after operation immediately and at the last follow-up compared with those before operation $(P<0.05)$, which shows that CICPS is a safe and effective treatment for patients with HVCFs. Additionally, there are no significant differences between VAS and ODI scores immediately after operation and at the final follow-up more than 6 months $(P>0.05)$. The result shows that satisfactory mid-term clinical outcomes can be achieved in this group. Singh $V$ et al. [29] did a systematic analysis for PMMA-augmented cannulated pedicle screw. Their results summarized from published studies indicated that the average VAS score before operation was 8.4 (range 8-9.2) compared to 2.3 (range 1.42-4.8) at the last follow up. The average improvement ODI for assessment of functional recovery was 42.1 . In this study, VAS score before operation was 8.3 compared with 2.2 at last follow-up and ODI improved from 30.5 to 75.5 . These results show that CICPS has a great improvement in pain relief and function recovery similar to previous series.

PMMA leakage is a focus point to surgeons because neurological or spinal cord damage may cause by the situation $[12,18,30]$. Traditionally, PMMA is injected into the preset nail tunnel using a PVP device during laddering period, and then the screw is inserted [25]. The challenges of this procedure lie in uncontrollable diffusion of PMMA during the screw insertion process. Even if PMMA is removed 
immediately when the spinal canal leakage occurs, these surgical procedures will lead to the time window of PMMA injection being exceeded. Therefore, we chose to further research CICPS instead of traditional injection method. The advantage of this method is that screws are left in situ and monitored by intraoperative X-ray when PMMA injection, which have less possibility of PMMA leakage due to pressurization. Moreover, the design of side holes located at distal third of screw also play an important role to avoid PMMA leakage [8-10].

Authors used CICPSs in 18 patients with HVCFs and osteoporosis. 3 screws in 2 patients $(3 / 40,7.5 \%)$ were observed PMMA leakage, but without any neurological symptoms. Wuisman et al. [31] reported four out of 49 screws (8.2\%) had PMMA leakage into spinal canal using traditional technique and removed PMMA by laminectomy and dura manipulation. Janssen et al. [32] reported that the incidence of asymptomatic PMMA leakage was $66.7 \%$ and that of symptoms was $5.5 \%$, in $1.2 \%$ of which needed to remove the PMMA and screws. Martı́n-Ferna'ndez et al. [33] observed a $62.3 \%$ incidence of spinal leakage in 313 patients. $1.55 \%$ of the cases had symptoms such as radicular pain of lower limbs and transient sensorimotor deficit. In brief, PMMA leakage presented no symptoms or only mild symptoms in most studies and the leakage rate showed a big difference, which reason might be related to the small sample size or different screw design in different studies.

In our series, the loss of kyphosis cobb angle increased significantly $(P<0.05)$ comparing the date of last follow-up with that of post-operation, while the vertebral height was no significant change $(P>0.05)$ and the screw loosening was not observed. The reason for this phenomenon can be explained by physiological progress. Studies have shown that kyphosis progressed an average 7 degree over 15 years in healthy women, especially between age of 50 and 59 [34, 35]. In thoracolumbar burst fracture following internal fixation without PMMA augmentation, progressive kyphosis was also observed [36]. Therefore, trauma-related disc apoptosis or osteoporosis process [37-39] may play important roles in kyphosis progression spite of PMMA augmentation or not.

HVCFs usually occurs in elderly patients over 50 years. These patients may have had many comorbidities and severe osteoporosis, which resulted in complex surgical procedures might not be tolerated. In one study of Huang et al [30], they observed the clinical outcomes of traditional PMMA augmentation technology in 28 cases of Kummell's disease. The operation time, blood loss and hospitalization time were $115.9 \pm 10.0$ minutes, $214.3 \pm 17.5 \mathrm{ml}$ and $7.6 \pm 1.3$ days respectively. While in this study, these data were $165 \pm 23$ minutes, $206 \pm 75 \mathrm{ml}$ and $12.5 \pm 3.5$ days. Furthermore, 3 patients had asymptomatic PMMA leakage, one developed a urinary tract infection, one contracted pneumonia, and one experienced delayed wound healing. There was no loosening at the final follow-up. We cannot draw any better conclusions by compared these data with our findings. However, the absolute values of the data between CICPS and the traditional PMMA augmentation technology only have small differences, which may be related to the technical proficiency of surgeons and postoperative management procedures.

This study has several limitations. Firstly, this study is not a randomized controlled study, which contributed to a low level of evidence. Secondly, the sample size is small and the follow-up time is short, 
thus a study with more patients and longer follow-up duration is required to further confirm our results. Thirdly, since the safety and effectiveness of CICPS have been biomechanically evaluated before [8], here only clinical evaluation was performed. Finally, standard application protocol of CICPS should be developed to avoid the catastrophic consequences of excessive application, which will be our future research focus.

\section{Conclusion}

In conclusion, CICPS using PMMA augmentation maintains their functional outcomes after internal fixation at latest follow-up in spite of kyphosis progression. The incidence of asymptomatic PMMA leakage, screw loosening and complications is not higher than that of previous reports. CICPS may be a clinically safe and effective technology which is used to augment pedicle screws in HVCFs with osteoporosis. This study could provide evidence-based basis for developing the guidelines of the cannulated pedicle screw application, especially in HVCFs with osteoporosis.

\section{Abbreviations}

HVCF

High-energy vertebral compression fracture;CICPS:Cement-injectable cannulated pedicle screw;PMMA:Polymethylmethacrylate; VAS:Visual analog scale; ODI:Oswestry disability index;PVP:Percutaneous vertebroplasty; PKP:Percutaneous kyphoplasty; VCF:Vertebral compression fracture

\section{Declarations}

\section{Acknowledgments}

We acknowledge the nursing staff from the Department of Orthopedic Surgery, Southwest Hospital of Army Medical University for their support during the study period.

\section{Authors' contributions}

YYL and JX conducted the experiments and wrote the manuscript, $X Y, M Y L, Z W$ and LH collected and analyzed the data. PL and FD supervised the project. All authors read and approved the final manuscript.

\section{Funding}

This work was supported by grants from the National Natural Science Foundation of China (No. 81902257) and our University Project of Medical Elite Training (2018XLC2024 and 2019CXLCB015).

\section{Availability of data and materials}


The datasets used and/or analysed during the current study are available from the corresponding author on reasonable request.

\section{Ethics approval and consent to participate}

The study was approved by the Daping Hospital ethics committee (IRB, 2019149).

\section{Consent for publication}

Informed consent for publication was obtained from all participants.

\section{Competing interests}

All authors declare no conflicts of interest.

\section{Authors' information}

Full list of author information is available at the first of the article

\section{References}

1. Sozen T, Ozisik L, Basaran NC. An overview and management of osteoporosis. European journal of rheumatology. 2017;4(1):46-56.

2. Porter JL, Bhimji SS. Osteoporosis. Treasure Island.FL: StatPearls Publishing; 2017. Available at:https://www.ncbi.nlm.nih.gov/books/NBK441901/.Accessed October 20, 2017.

3. Lee YK,Jang S,Jang S, Lee H J,Park C,Ha YC, et al. Mortality after vertebral fracture in Korea: analysis of the National Claim Registry. Osteoporosis international : a journal established as result of cooperation between the European Foundation for Osteoporosis and the National Osteoporosis Foundation of the USA. 2012;23(7):1859-1865.

4. Mayumi S,Naoto E,Takeo O,Dai M,Yujiro O,Kanta I, et al. Incidence of osteoporotic fractures in Sado, Japan in 2010. Journal of bone and mineral metabolism. 2014;32(2):200-205.

5. Kong LD, Wang P, Wang LF, Shen Y, Shang ZK, Meng LC. Comparison of vertebroplasty and kyphoplasty in the treatment of osteoporotic vertebral compression fractures with intravertebral clefts. European journal of orthopaedic surgery \& traumatology : orthopedie traumatologie. 2014;24 Suppl 1:S201-208.

6. Hartmann F, Gercek E, Leiner L, Rommens PM. Kyphoplasty as an alternative treatment of traumatic thoracolumbar burst fractures Magerl type A3. Injury. 2012;43(4):409-415.

7. Patel V, Issever AS, Burghardt A, Laib A, Ries M, Majumdar S. MicroCT evaluation of normal and osteoarthritic bone structure in human knee specimens. Journal of orthopaedic research : official publication of the Orthopaedic Research Society. 2003;21(1):6-13.

8. Liu Y, Xu J, Sun D, Luo F, Zhang Z, Dai F. Biomechanical and finite element analyses of bone cementInjectable cannulated pedicle screw fixation in osteoporotic bone. Journal of biomedical materials 
research Part B, Applied biomaterials. 2016;104(5):960-967.

9. Dai F,Liu Y,Zhang F,Sun D,Luo F,Zhang Z, et al. Surgical treatment of the osteoporotic spine with bone cement-injectable cannulated pedicle screw fixation: technical description and preliminary application in 43 patients. Clinics. 2015;70(2):114-119.

10. Rong Z, Zhang F, Xiao J, Wang Z, Luo F, Zhang Z, et al. Application of Cement-Injectable Cannulated Pedicle Screw in Treatment of Osteoporotic Thoracolumbar Vertebral Compression Fracture (AO Type A): A Retrospective Study of 28 Cases. World neurosurgery. 2018;120:e247-e258.

11. Lowe TG, Tahernia AD, O'Brien MF, Smith DA. Unilateral transforaminal posterior lumbar interbody fusion (TLIF): indications, technique, and 2-year results. Journal of spinal disorders \& techniques. 2002;15(1):31-38.

12. Wu JC, Huang WC, Tsai HW, Ko CC, Wu CL,Tu TH, et al. Pedicle screw loosening in dynamic stabilization: incidence, risk, and outcome in 126 patients. Neurosurgical focus. 2011;31(4):E9.

13. Ko CC, Tsai HW, Huang WC ,Wu JC,Chen YC,Shih YH, et al. Screw loosening in the Dynesys stabilization system: radiographic evidence and effect on outcomes. Neurosurgical focus. 2010;28(6):E10.

14. Dakhil-Jerew F, Jadeja H, Cohen A, Shepperd JA. Inter-observer reliability of detecting Dynesys pedicle screw using plain X-rays: a study on 50 post-operative patients. European spine journal : official publication of the European Spine Society, the European Spinal Deformity Society, and the European Section of the Cervical Spine Research Society. 2009;18(10):1486-1493.

15. Hsieh MK, Chen LH, Chen WJ. Current concepts of percutaneous balloon kyphoplasty for the treatment of osteoporotic vertebral compression fractures: evidence-based review. Biomedical journal. 2013;36(4):154-161.

16. Tang H, Zhao JD, Li Y, Dai H. Efficacy of percutaneous kyphoplasty in treating osteoporotic multithoracolumbar vertebral compression fractures. Orthopedics. 2010;33(12):885.

17. Gan M, Yang H, Zhou F, Zou J,Wang G,Mei X, et al. Kyphoplasty for the treatment of painful osteoporotic thoracolumbar burst fractures. Orthopedics. 2010;33(2):88-92.

18. Yang H, Pan J, Sun Z, Mei X, Yang Y. Percutaneous augmented instrumentation of unstable thoracolumbar burst fractures: our experience in preventing cement leakage. European spine journal : official publication of the European Spine Society, the European Spinal Deformity Society, and the European Section of the Cervical Spine Research Society. 2012;21(7):1410-1412; author reply 1413.

19. McLain RF, Burkus JK, Benson DR. Segmental instrumentation for thoracic and thoracolumbar fractures: prospective analysis of construct survival and five-year follow-up. The spine journal: official journal of the North American Spine Society. 2001;1(5):310-323.

20. Lonstein JE, Denis F, Perra JH, Pinto MR, Smith MD, Winter RB. Complications associated with pedicle screws. The Journal of bone and joint surgery American volume. 1999;81(11):1519-1528.

21. Frankel BM, Jones T, Wang C. Segmental polymethylmethacrylate-augmented pedicle screw fixation in patients with bone softening caused by osteoporosis and metastatic tumor involvement: a clinical evaluation. Neurosurgery. 2007;61:531-537; discussion 537-538. 
22. Fransen P. Increasing pedicle screw anchoring in the osteoporotic spine by cement injection through the implant. Technical note and report of three cases. J Neurosurg Spine. 2007;7:366-369.

23. Jang SH, Lee JH, Cho JY, Lee HY, Lee SH. The efficacy of hydroxyapatite for screw augmentation in osteoporotic patients. Neurol Med Chir. 2013;53:875-881.

24. Chandra Vemula VR, Prasad BC, Jagadeesh MA, Vuttarkar J, Akula SK. Minimally invasive transforaminal lumbar interbody fusion using bone cement-augmented pedicle screws for lumbar spondylolisthesis in patients with osteoporosis. Case series and review of literature. Neurol India. 2018;66:118-125.

25. Sawakami K, Yamazaki A, Ishikawa S, Ito T, Watanabe K, Endo N. Polymethylmethacrylate augmentation of pedicle screws increases the initial fixation in osteoporotic spine patients. J Spinal Disord Tech. 2012;25:E28-E35.

26. Park SJ, Kim HS, Lee SK, Kim SK. Bone cement-augmented percutaneous short segment fixation: an effective treatment for Kummell's disease? J Korean Neurosurg Soc. 2015;58:54-59.

27. Cho Y. Posterior vertebrectomy and circumferential fusion for the treatment of advanced thoracolumbar Kummell disease with neurologic deficit. Asian Spine J. 2017;11:634-640.

28. Liu Y, Xiao J, Yin X,Liu M;Zhao J;Liu P ,et al. Clinical efficacy of bone cement-injectable cannulated pedicle screw short segment fixation for lumbar spondylolisthesis with osteoporosise. Sci Rep. 2020;10(1):3929

29. Singh V, Mahajan R, Das K, Chhabra HS, Rustagi T. Surgical trend analysis for use of cement augmented pedicle screws in osteoporosis of spine: a systematic review (2000-2017). Global Spine J. 2019;9:783-795.

30. Rohith Kumar R. Ashok Kumar S, Periyasami Bhuvana K. Preparation and evaluation of bioactivity of porous bioglass tablets for bone tissue regeneration. Sci Medicine J. 2019;1(3):112-123.

31. Wuisman PI, Van Dijk M, Staal H, Van Royen BJ. Augmentation of (pedicle) screws with calcium apatite cement in patients with severe progressive osteoporotic spinal deformities: an innovative technique. Eur Spine J. 2000;9:528-533.

32. Janssen I, Ryang YM, Gempt J, Bette S; Gerhardt J,Jan S, et al. Risk of cement leakage and pulmonary embolism by bone cement-augmented pedicle screw fixation of the thoracolumbar spine. Spine J. 2017;2:837-844.

33. Martín-Ferna'ndez M, López-Herradón A, Piñera AR, Tomé-Bermejo F, Duart JM., Vlad MD,et al. Potential risks of using cement-augmented screws for spinal fusion in patients with low bone quality. Spine J. 2017;17:1192-1199.

34. Kado DM, Miller-Martinez D, Lui LY, Cawthon P, Katzman WB, Hillier TA ,et al. Hyperkyphosis, kyphosis progression, and risk of non-spine fractures in older community dwelling women: the study of osteoporotic fractures (SOF). J Bone Miner Res. 2014;29:2210-2216.

35. Ball JM, Cagle P, Johnson BE, Lucasey C, Lukert BP. Spinal extension exercises prevent natural progression of kyphosis. Osteoporos Int. 2009;20:481-489. 
36. Lakshmanan P, Jones A, Mehta J, Ahuja S, Davies PR, Howes JP. Recurrence of kyphosis and its functional implications after surgical stabilization of dorsolumbar unstable burst fractures. Spine J. 2009;9:1003-1009.

37. Lin HH, Chang MC, Wang ST, Liu CL, Chou PH. The fates of pedicle screws and functional outcomes in a geriatric population following polymethylmethacrylate augmentation fixation for the osteoporotic thoracolumbar and lumbar burst fractures with mean ninety five month follow-up. International Orthopaedics. 2018;42(6):1313-1320.

38. Zhang SJ. Clark M, Liu XL. The effects of bio-inspired electromagnetic fields on healthy enhancement with case studies. Emerging Science J. 2019;3(6):369-381.

39. Swati P, Nikita S. Effect of body positions on quadriceps angle measurement. Sci Medicine J. 2019;1(1):20-2.

\section{Figures}




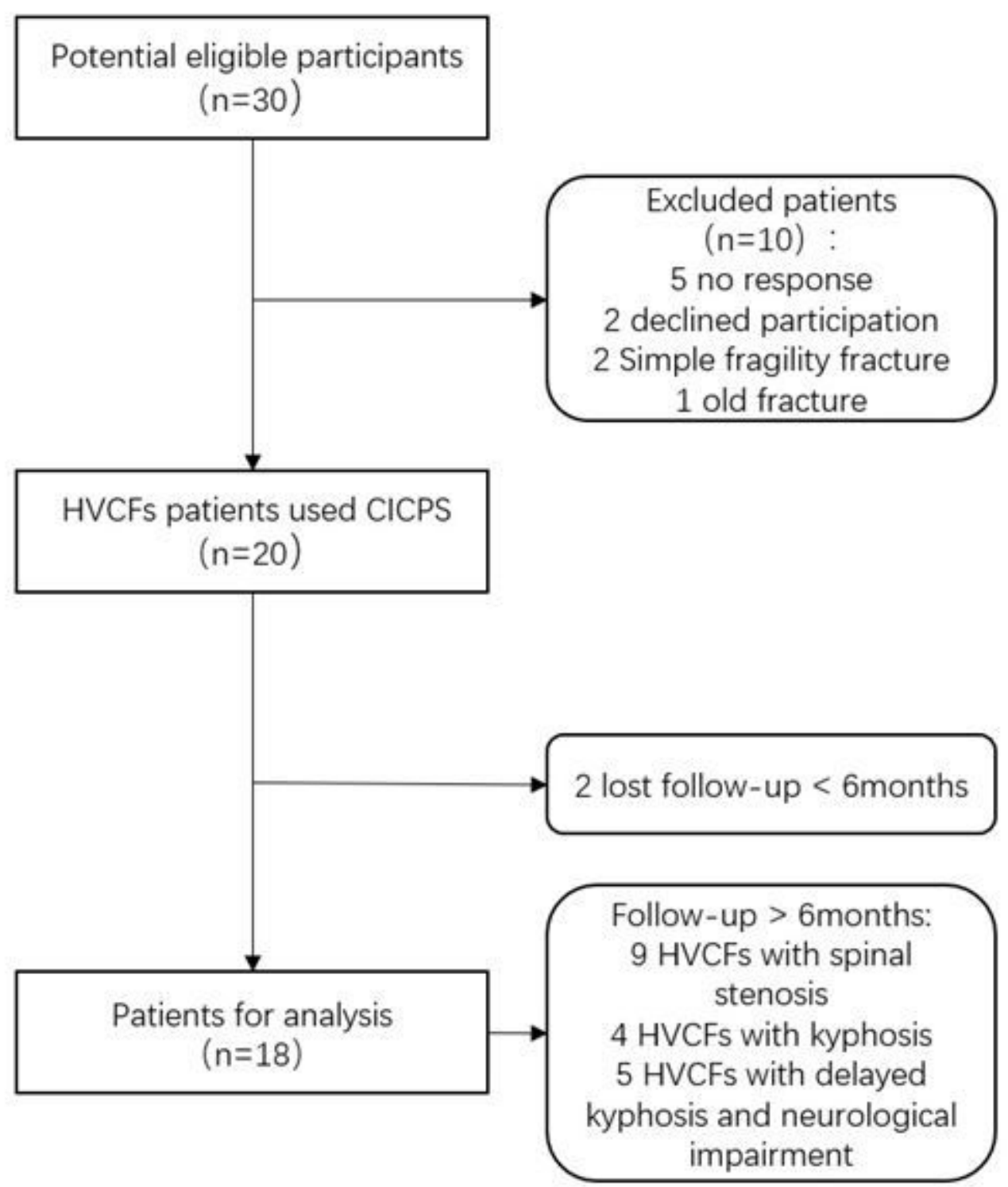

Figure 1

Flow chart of the patients included. 


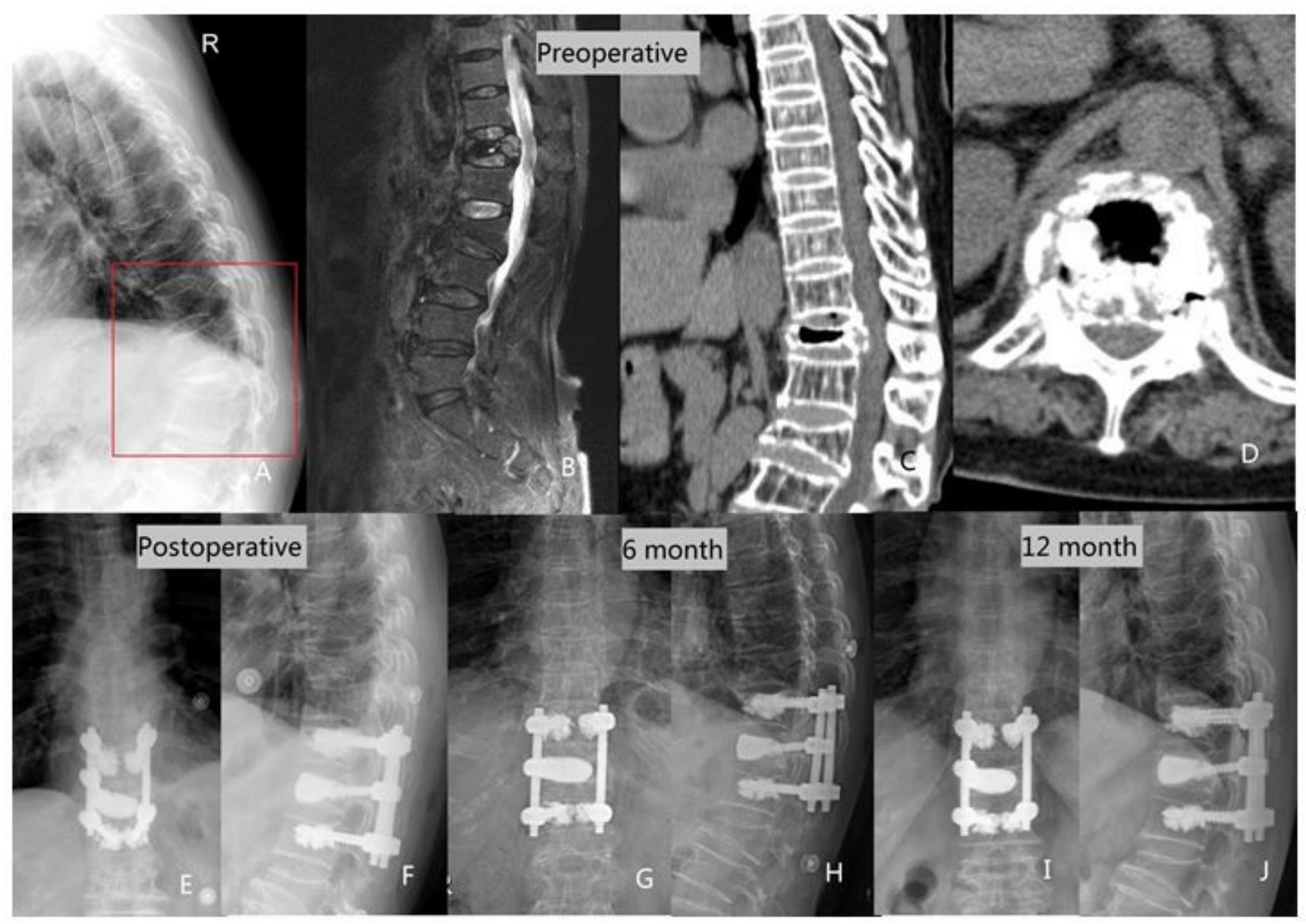

Figure 2

A-D, the preoperative X-ray, MRI and CT indicated T12 HVCF with kyphosis. E-F, the postoperative X-ray indicated that the height of T12 was restored and the kyphosis was corrected. G-J, there was no significant loss of vertebral height and kyphosis angle at 6 and 12-month follow-up. 


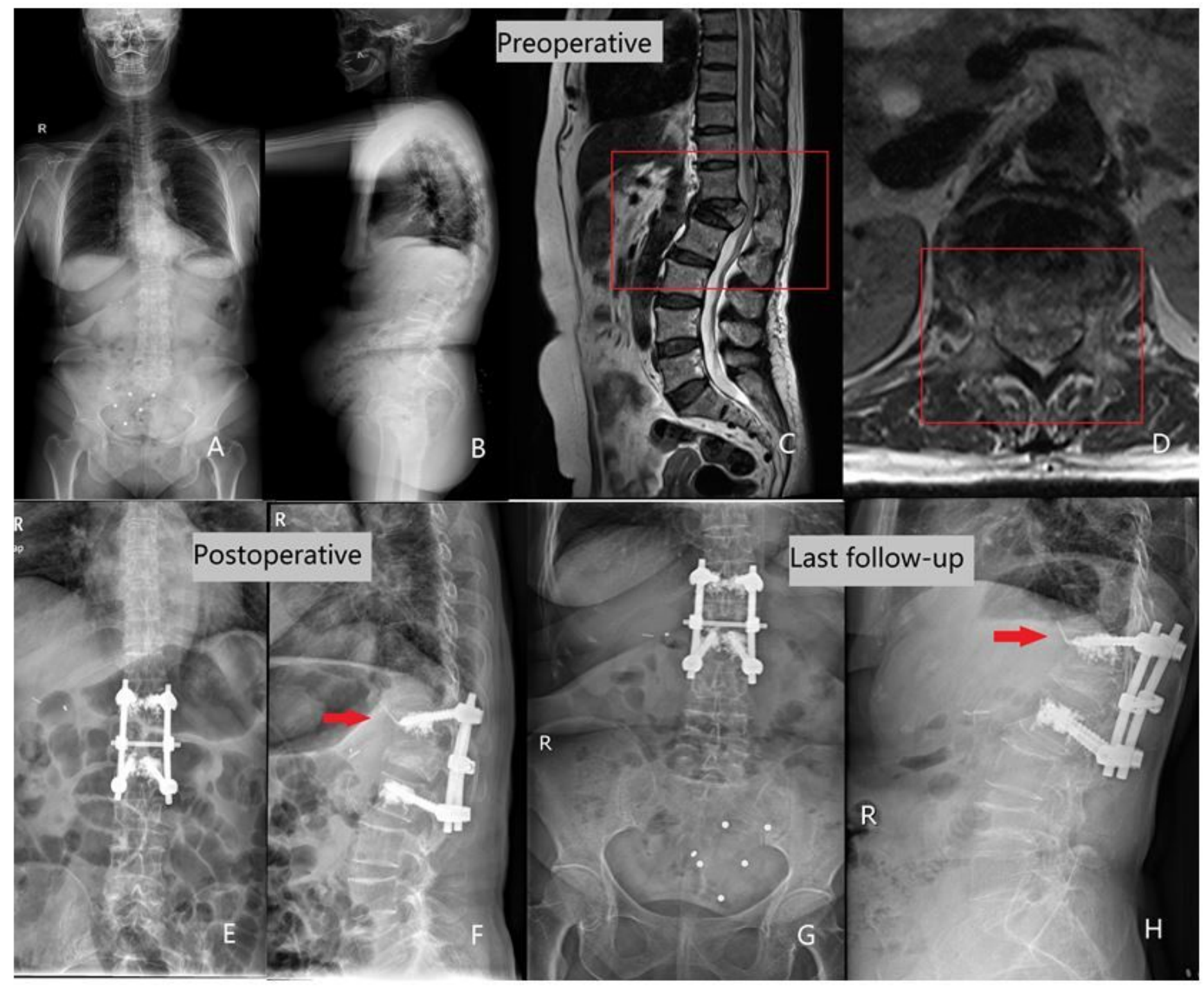

Figure 3

A-D, the preoperative X-ray and MRI indicated L1 HVCF with kyphosis and spinal stenosis. E-F, the postoperative X-ray revealed partial recovery of the height of $\mathrm{L} 1$ and the kyphosis angle. G-H, there was minimal loss of kyphosis angle at18-month follow-up, but vertebral height was maintained; the red arrow indicated PMMA leakage into the venous plexus. 